\title{
Motor response detection using fiber optics during functional magnetic resonance imaging
}

\author{
JÖRG MEINHARDT and JÜRGEN MÜLLER \\ University of Regensburg, Regensburg, Germany
}

\begin{abstract}
Data acquisition during functional magnetic resonance imaging and magnetoencephalography studies is easily disturbed by metallic and magnetic devices. Nevertheless, recording motor responses during many neuropsychological studies is essential. In this paper, we describe the design and construction of a nonmetallic, nonmagnetic motor response recording device that uses fiber optics to obtain interference-free data.
\end{abstract}

Functional magnetic resonance imaging (fMRI) is a method used to study brain activity during task performance noninvasively. Most elaborated experimental designs require the recording of additional behavioral data in order to link brain activity with different response categories during scanning. It is difficult to obtain motor response data during fMRI scanning, since electronic devices are compromised by the magnetic field within the scanner and vice versa. In detail, MRI relies on the energy released by protons in atomic nuclei as they return to their resting state after having been temporarily perturbed. Protons precess as they return to rest, which produces a characteristic energy signal. The production of a signal strong enough to be recorded requires that a large number of protons return to their resting state in coherent fashion. This produces an aggregate signal that can be detected by the MRI scanning device. The large magnet used in MRI scanners produces a strong ambient magnetic field, which ensures that a large number of protons within range of the field are in a similar resting state. To generate an image, the scanner produces a brief energy pulse at radio frequency within the plane to be imaged, which momentarily perturbs the protons within that plane. It then records the energy that is released as those protons precess back to their original states (cf. Cohen, Noll, \& Schneider, 1993; Krishnan \& MacFall, 1997; Lewis, 1996).

fMRI challenges the design of experimental equipment. The static magnetic field itself and the radio-frequency signals of the MRI scanner place constraints on the physical characteristics of the hardware used for motor re-

We gratefully acknowledge the assistance of Josef Bernhardt and Herbert Tischhöfer for technical support. None of the authors has any commercial or financial interest in any hardware product that is referred to in the article. The authors have contributed equally. The order of authors is alphabetical. J. Müller is in the Department of Psychiatry at Regensburg. Send correspondence to J. Meinhardt, Institute of Psychology, University of Regensburg, Universitätsstrasse 31, D-93040 Regensburg, Germany (e-mail: joerg.meinhardt@ psychologie.uni-regensburg.de). sponse registration. Ferromagnetic materials cannot be brought into close proximity with the scanner, and electronic devices must not produce a significant amount of radio-frequency noise, or must be shielded, which is often difficult to do.

Cable movements caused by vibrations of the scanner, head actions, or small body motions (due to heart beats or breathing) lead to movement of the wires. Moving wires within a magnetic field induce voltage in the wire that (1) overlap with the MRI signal and worsen the signal-tonoise ratio of the MRI, and (2) might interfere with the signal transformation of the response registration device and thus interrupt the acquisition of the response data. The amplitude of these artifacts is expected to increase with the field strength of the scanner, as has been shown in recent studies in which $\mathrm{fMRI}$ and EEG were applied simultaneously (Allen, Polizzi, Krakow, Fish, \& Lemieux, 1998).

\section{Data Registration by Opto-Switch During fMRI Studies}

This problem is of major relevance in neuroimaging research requiring response data from subjects. To connect functional imaging study designs and different neuropsychological approaches, behavioral data of the subjects have to be registered simultaneously with the fMRI acquisition. For studies in which subject responses must be recorded, we have constructed a fiber-optic keypad that transfers response data via a fiber-optic cable to a transceiver, which, in turn, is connected to a microcomputer via a parallel input device (for example). An overview of the Opto-Switch is given in Figure 1. The Opto-Switch comprises a response pad, fiber-optic cables, and a transceiver. Thus, there is no alternating electrical signal within the response device that might produce RF interference. We describe the design and construction of a nonmetallic, nonmagnetic keypad used for registering the subject's motor response during MRI scans and related methods working on lighting wire. 


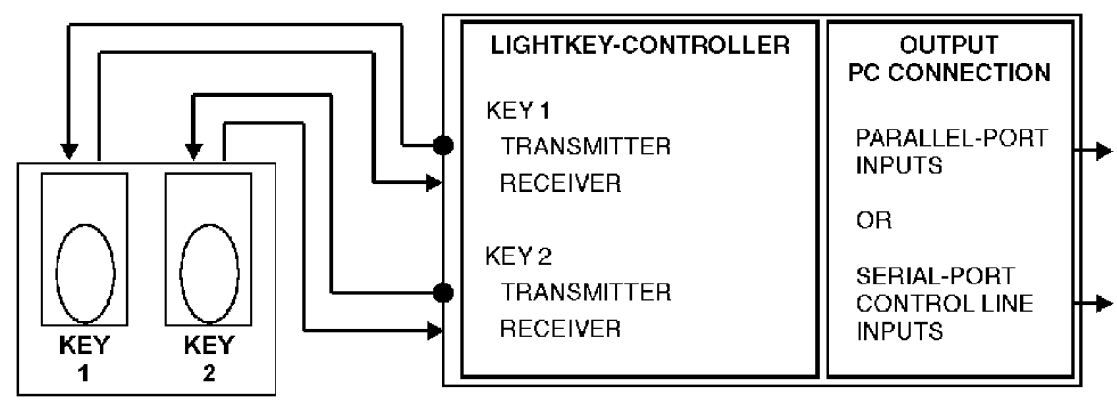

Figure 1. Overview of the construction of the MRI Opto-Switch. The device consists of a response pad (inside the magnetic field), a transceiver (outside the magnetic field), and a fiber-optic cable. The transmitter (TOTX173; see Figure 3) provides a light source that passes through the fiber-optic cable into the switch. The light beam may be interrupted before it is picked up by the fiber-optic cable to run the light back to the receiver (TORX173; see Figure 3). Note-The response pad and the transceiver are connected only via fiber-optic components. Thus only light signals and nonmetallic materials pass through the field of the MRI.

The response data registration device works as follows: Outside the critical magnetic field, a customary fiber-optic transmitting module TOTX173 (Toshiba) sends out a light signal. This light signal is transferred by the fiber-optic cable to a metal free response key, which can be located in the magnetic field. Inside the response key, the light beam is interrupted or closed, due to the response of the subjects (Figure 2). The output is then transferred by fiber-optic cables back to the fiber-optic receiving module TORX173 (Toshiba) located outside of the critical magnetic field. The fiber-optic cable is connected to the transmitter socket (TOTX173) and receiver socket (TORX173) with TOCP155 (Toshiba) plugs. In this manner, only fiber-optic components pass through the magnetic field of the MRI. The voltage outputs of the two TORX173 devices can be connected to the inputs of a printer port, or (via a MAX220 level converter, Maxim Integrated Products, Inc.) to the control lines (CTS, clear-to-send; and RI, ring indicator) of a serial port. Thus, the Opto-Switch can report switch closures to the controlling program through either the parallel or the serial port.

\section{Construction of the Opto-Switch}

The Opto-Switch consists of a key lever that interrupts the light beam if pressed. The resilience is produced by a spring made of synthetic material (e.g. silicone) or a rubber ring. The prototype illustrated in Figure 2 measures $5.5 \times 2.5 \times 2.0 \mathrm{~cm}$. The distance that the light beam has to bridge is $3 \mathrm{~mm}$. Every key is supplied by its own transceiver. The light signal is converted into an electric signal by the transceiver and can thus be read by PC for example via a parallel interface (as is shown in Figure 3). The hardware components of the Opto-Switch allow for the registering of reaction data with a precision of milliseconds. The effective response time, however, depends on computer hard- and software components processing the data.

\section{Conclusion}

The Opto-Switch permits detection of motor responses during experiments that use fMRI. Acquiring fMRI data does not interfere with response detection or vice versa. The Opto-Switch can be constructed easily and at low cost.

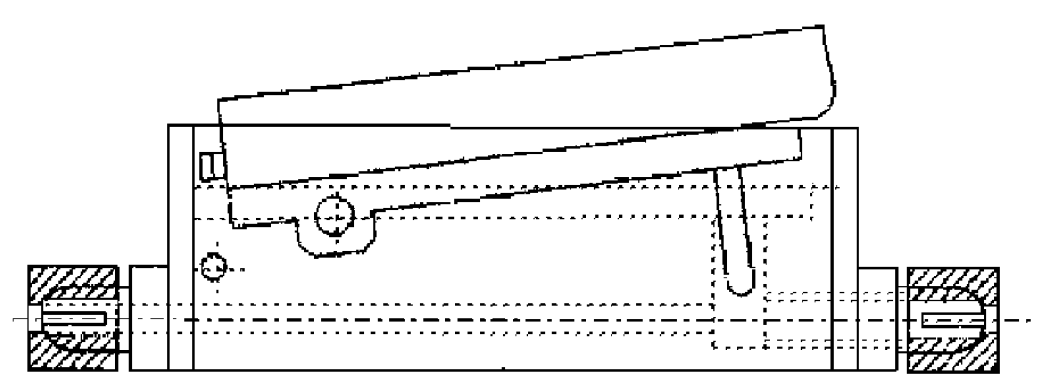

Figure 2. Construction of the nonmetallic Opto-Switch response pad: lateral view. 


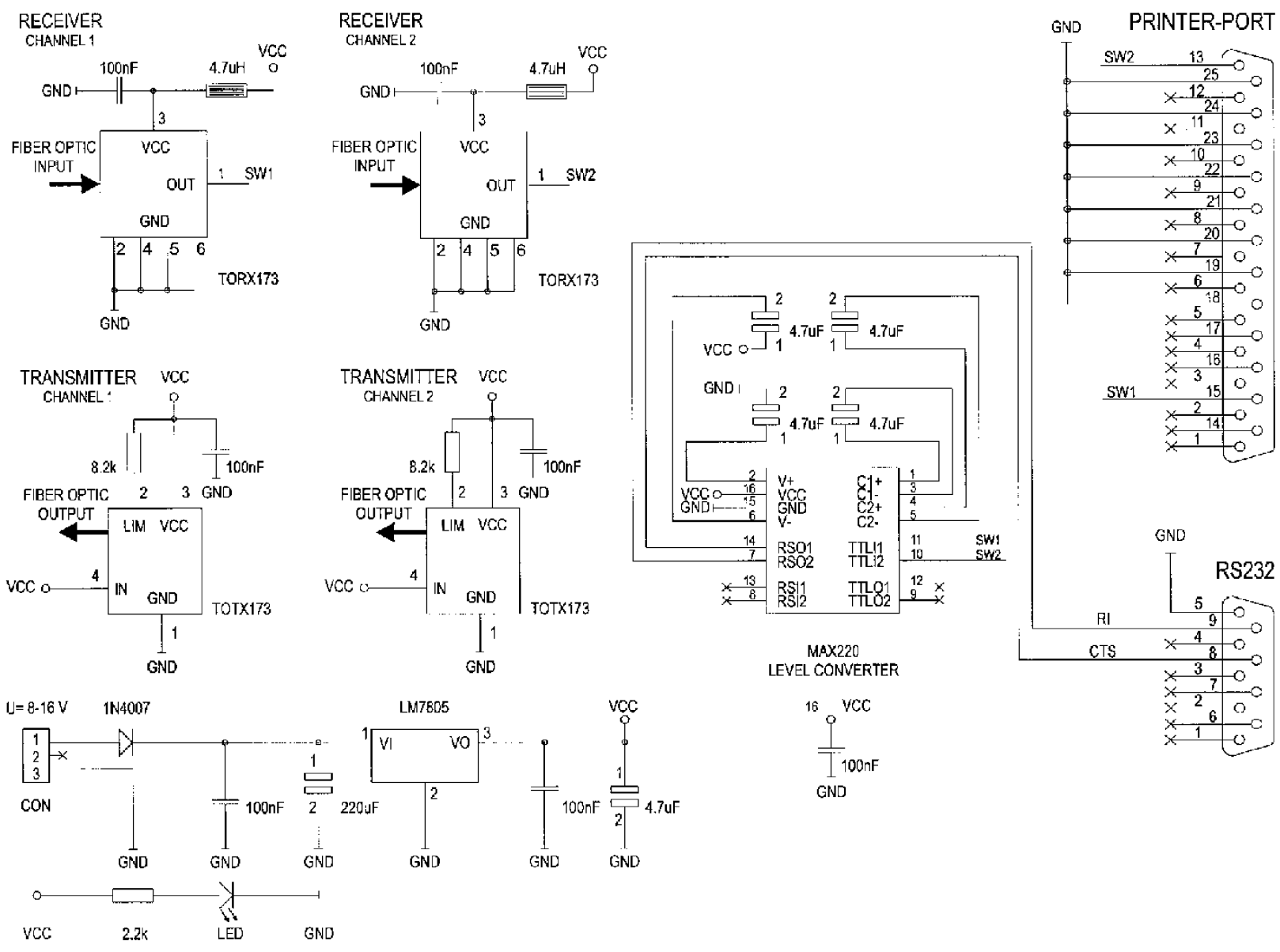

Figure 3. Wiring diagram of the opto-electric transceiver for two keys and of an output device (serial or parallel connector). The input is held high on the TOTX173 transmitters, which provide a light source that passes through the fiber-optic cable into the switch. The light beam may be interrupted before it is picked up by the fiber-optic cable to run the light back to the receiver (depicted in Figure 1). In order for Response 1 or Response 2 to be detected by the computer, the output of the TORX173 can be connected to an input line of a printer port or (via a MAX220 level converter) to the CTS or RI control input line of an RS232 serial port. Note-The serial port is used for parallel data processing through control lines CTS and RI.

\section{REFERENCES}

Allen, P. J., Polizzi, G., Krakow, K., Fish, D. R., \& Lemieux, L. (1998). Identification of EEG events in the MR scanner: The problem of pulse artifacts and a method for its subtraction. Neuroimage, 8, 229-239.

Cohen J. D., Noll, D. C., \& Schneider, W. (1993). Functional magnetic resonance imaging: Overview and methods for psychological research. Behavior Research Methods, Instruments, \& Computers, 25, 101-113.

Krishnan K. R. R., \& MaCFall, J. R. (1997). Basic principles of mag- netic resonance imaging. In K. R. R. Krishnan \& P. M. Doraiswamy (Eds.), Brain imaging in clinical psychiatry (pp. 1-11). New York: Dekker.

LEwIS, S. W. (1996). Functional brain imaging. In S. Lewis \& N. Higgins (Eds.), Functional brain imaging in psychiatry (pp. 108-115). Cambridge, U.K.: Blackwell.

(Manuscript received March 19, 2001; revision accepted for publication August 5, 2001.) 